\title{
ON THE STRUCTURE OF RIEMANNIAN MANIFOLDS OF ALMOST NONNEGATIVE RICCI CURVATURE
}

\author{
GABJIN YUN
}

Received 14 November 2002

\begin{abstract}
We study the structure of manifolds with almost nonnegative Ricci curvature. We prove a compact Riemannian manifold with bounded curvature, diameter bounded from above, and Ricci curvature bounded from below by an almost nonnegative real number such that the first Betti number having codimension two is an infranilmanifold or a finite cover is a sphere bundle over a torus. Furthermore, if we assume the Ricci curvature is bounded and volume is bounded from below, then the manifold must be an infranilmanifold.
\end{abstract}

2000 Mathematics Subject Classification: 53C20, 53C23.

1. Introduction. In this paper, we will consider a class of compact $n$-dimensional Riemannian manifolds $(M, g)$ satisfying

$$
\left|K_{g}\right| \leq \Lambda, \quad \operatorname{diam}(M) \leq D, \quad \operatorname{Ric}(M) \geq-\epsilon,
$$

where $K_{\mathfrak{g}}$, $\operatorname{diam}(M)$, and $\operatorname{Ric}(M)$ denote the sectional curvature, diameter, and Ricci curvature, respectively, of a Riemannian manifold $(M, g)$, while $D$ and $\Lambda$ are positive real numbers and $\epsilon$ is usually a sufficiently small positive real number.

In [8], Gromov proved that there is an $\epsilon>0$ depending only on $n$ and a given constant $D>0$ such that if $\operatorname{diam}(M) \leq D$ and $\operatorname{Ric}(M) \geq-\epsilon$, then the first Betti number of $M$, $b_{1}(M)$, is bounded by $n$, that is, $b_{1}(M) \leq n$. Gallot [6] also gave an analytic proof for this. In [12], Yamaguchi has shown that if a Riemannian manifold $(M, g)$ satisfies the conditions (1.1), then there is a smooth fibration

$$
F \rightarrow M \longrightarrow T^{b_{1}(M)}
$$

where $T^{b_{1}(M)}$ is the $b_{1}(M)$-dimensional torus. This implies that if $b_{1}(M)=n$, then $M$ is diffeomorphic to the $n$-dimensional torus $T^{n}$ and if $b_{1}(M)=n-1$, then $M$ is diffeomorphic to an infranilmanifold, that is, a finite covering space of $M$ is a quotient of a simply connected nilpotent Lie group by a lattice.

In this paper, we study the structure of Riemannian manifolds satisfying the conditions (1.1) and whose first Betti number is $n-2$ or $n-3$. In case the first Betti number is $n-2$, there are at least two known families of manifolds with metrics satisfying (1.1): infranilmanifolds and compact quotients of the product space $M=S^{2} \times \mathbb{R}^{n-2}$. We will see below that there are only such cases if the first Betti number $b_{1}(M)=n-2$. In case $b_{1}(M)=n-3$, since there is lack of examples, we only consider manifolds of dimension 4 . 
Throughout this paper, the dimension of manifolds is denoted by $n$ unless otherwise stated.

2. Almost nonnegative Ricci curvature and the first Betti number. In this section, we consider Riemannian manifolds $(M, g)$ satisfying the conditions (1.1) with restriction on the first Betti number $b_{1}(M)$. First of all, we would like to mention a theorem due to Cheeger and Colding, which is crucially used in the proofs of our results. This theorem, conjectured originally by Gromov, says that the fundamental groups of a class of Riemannian manifolds with almost nonnegative Ricci curvature are almost nilpotent.

THEOREM 2.1 [3]. Given a positive integer $n$ and $D>0$, there exists $\epsilon=\epsilon(n, D)>0$ such that if $\left(M^{n}, g\right)$ is a compact Riemannian n-manifold satisfying

$$
\operatorname{diam}(M) \leq D, \quad \operatorname{Ric}(M) \geq-\epsilon,
$$

then the fundamental group $\pi_{1}(M)$ is almost nilpotent, that is, it contains a nilpotent subgroup of finite index.

Now we prove a structure theorem for manifolds satisfying the conditions (1.1) with $b_{1}(M)=n-2$.

Proposition 2.2. Given $\Lambda>0, D>0$, and a natural number $n$, there exists $\epsilon=$ $\epsilon(\Lambda, D, n)>0$ such that if $\left(M^{n}, g\right)$ is a compact Riemannian $n$-manifold satisfying

$$
\left|K_{\mathfrak{g}}\right| \leq \Lambda, \quad \operatorname{diam}(M) \leq D, \quad \operatorname{Ric}(M) \geq-\epsilon, \quad b_{1}(M)=n-2,
$$

then $M$ is a fiber bundle over $T^{n-2}$ with the property that a finite cover of the fiber is diffeomorphic to $T^{2}$ or $S^{2}$.

Proof. Choose $\epsilon>0$ sufficiently small so that the properties in [12] and Theorem 2.1 hold. First note that, due to [12], $M$ is a fiber bundle over $T^{n-2}$, that is, there is a fibration

$$
F \longrightarrow M \longrightarrow T^{n-2}
$$

where $T^{n-2}$ is the $(n-2)$-dimensional torus.

By the uniformization theorem, a finite cover $\hat{F}$ of $F$ is diffeomorphic to $S^{2}, T^{2}$, or $\Sigma$, a surface of genus greater than or equal to 2 . We will show that $\hat{F}$ cannot be diffeomorphic to $\Sigma$. Assume $\hat{F}$ is diffeomorphic to $\Sigma$. It follows from (2.3) that there is an exact sequence of homotopy groups

$$
0=\pi_{2}\left(T^{n-2}\right) \longrightarrow \pi_{1}(F) \longrightarrow \pi_{1}(M) .
$$

Since $\pi_{1}(M)$ is almost nilpotent by Theorem 2.1, the sequence (2.4) shows that $\pi_{1}(F)$ is also almost nilpotent. However, since $\Sigma$ is a surface of genus greater than or equal to 2 , it is well known that $\pi_{1}(\Sigma)$ cannot be almost nilpotent. Hence the proof is complete.

Before going ahead, we state a basic algebraic lemma about a geometric group, which follows actually from [9]. 
LEMMA $2.3[9,14]$. Let $\Gamma$ be a finitely generated group of polynomial growth. Then it contains a torsion-free nilpotent subgroup of finite index.

A solvable group $\Gamma$ is called polycyclic if there is a subnormal series

$$
\Gamma=\Gamma_{0} \supset \Gamma_{1} \supset \cdots \supset \Gamma_{k}=\{e\},
$$

where factors $\Gamma_{i} / \Gamma_{i+1}$ are all infinite-cyclic and $e$ denotes the identity element in $\Gamma$. A solvable group is almost polycyclic if it contains a subgroup of finite index, which is polycyclic. The number of infinite cyclic factors is independent of the choice of finiteindex subgroup or subnormal series, and is called the Hirsch length of the group.

Now we prove our main theorem as an application of Proposition 2.2 by using Lemma 2.3 and Theorem 2.1

THEOREM 2.4. Given $\Lambda>0, D>0$, and a natural number $n$, there exists $\epsilon=$ $\epsilon(n, \Lambda, D)>0$ such that if $\left(M^{n}, g\right)$ is a compact Riemannian manifold satisfying (2.2), then $M$ is an infranilmanifold or a finite cover of $M$ is an $S^{2}$-bundle over $T^{n-2}$.

Proof. Choose $\epsilon>0$ sufficiently small so that Proposition 2.2 holds. Suppose $(M, g)$ is a Riemannian $n$-manifold satisfying (2.2). $M$ is a fiber bundle over $T^{n-2}$ with a fiber being a quotient of $S^{2}$ or $T^{2}$. It is enough to show that if the fiber is a quotient of $T^{2}$, then $M$ is an infranilmanifold. By Theorem 2.1 again, $\pi_{1}(M)$ is almost nilpotent. So, by Lemma $2.3, \pi_{1}(M)$ has a torsion-free nilpotent subgroup of finite index $\Gamma$. From the above fibration, we have an exact sequence of homotopy groups

$$
0 \longrightarrow K \longrightarrow \pi_{1}(M) \longrightarrow \mathbb{Z}^{n-2} \longrightarrow 0,
$$

where $K$ is isomorphic to $\mathbb{Z}^{2} \oplus H$ and $H$ is a finite group.

Note that the universal covering $\widetilde{M}$ of $M$ is diffeomorphic to $\mathbb{R}^{n}$ and $\Gamma$ has Hirsch length $n$. The nilpotent Malcev completion $N$ of $\Gamma$ can now be identified with $\widetilde{M}$. So, $\widetilde{M}$ is a simply connected nilpotent Lie group with a lattice subgroup $\Gamma$. This means that $M$ is an infranilmanifold.

REMARK 2.5. A converse of Theorem 2.4 holds, that is, any nilmanifold or any $S^{2}$ bundle over $T^{n-2}$ has Riemannian metrics which satisfy (2.2) for any $\epsilon$.

REMARK 2.6. In [4], 4-dimensional compact nilmanifolds with $b_{1}=2$ can be described explicitly.

Now we consider Riemannian manifolds of dimension 4 and the first Betti number $b_{1}(M)=1$. In case dimension $n=4$, it is notable that there are no 4-dimensional compact infranilmanifolds with $b_{1}(M)=1$ [11].

THEOREM 2.7. For given $\Lambda>0$ and $D>0$, there exists $\epsilon=\epsilon(\Lambda, D)>0$ such that if $\left(M^{4}, g\right)$ is a compact Riemannian 4-manifold satisfying

$$
\left|K_{g}\right| \leq \Lambda, \quad \operatorname{diam}(M) \leq D, \quad \operatorname{Ric}(M) \geq-\epsilon, \quad b_{1}(M)=1,
$$

then $M$ is a fibration over $S^{1}$ whose fiber is homotopic to a spherical space form $S^{3} / \Gamma$ for some finite subgroup $\Gamma$ acting on $S^{3}$. 
Proof. By [12], there exists an $\epsilon>0$ such that if $\left(M^{n}, g\right)$ is a closed Riemannian manifold satisfying (2.7), then $M$ is a fibration over $S^{1}$,

$$
F \longrightarrow M \longrightarrow S^{1}
$$

On the other hand, by Theorem $2.1, \pi_{1}(M)$ is almost nilpotent, and so it has a polynomial growth. It follows from Lemma 2.3 that $\pi_{1}(M)$ contains a torsion-free nilpotent subgroup $\Gamma$ of finite index. Since $b_{1}(M)=1, \Gamma$ is abelian, and so $\Gamma \cong \mathbb{Z}$. In fact, if $\Gamma$ is not abelian, then it contains a subgroup which is isomorphic to the Heisenberg group (see Remark 2.8), and so the growth of $\Gamma$ is at least 4 and $b_{1}(M) \geq 2$ (cf. [2, Section 7]).

Thus, $\pi_{1}(M) \cong \mathbb{Z} \oplus H$, where $H$ is a finite group and $\pi_{1}(F)$ is also finite group. Hence the universal cover $\widetilde{F}$ of $F$ is a compact simply connected 3-manifold, and so $\widetilde{F}$ is a homotopy 3-sphere, that is, $F$ is homotopic to $S^{3} / H$ for some finite group $H$ acting on $S^{3}$.

REMARK 2.8. In dimension $n \geq 5$, replacing the condition on the first Betti number by $b_{1}(M)=n-3$, Theorem 2.7 does not hold anymore. For example, let $N$ be the Heisenberg group

$$
N=\left\{\left(\begin{array}{ccc}
1 & x & z \\
0 & 1 & y \\
0 & 0 & 1
\end{array}\right) \mid x, y, z \in \mathbb{R}\right\}
$$

and $\Gamma$ its integer lattice. Then $M:=N / \Gamma$ is a compact orientable 3-dimensional nilmanifold. It is well known that $b_{1}(M)=2$ and $M$ is an $S^{1}$-bundle over $T^{2}$. For a given $\epsilon>0$, since $M$ is a nilmanifold, there is a metric $g_{\epsilon}$ such that

$$
\left|K_{g_{\epsilon}}\right| \leq 24 \epsilon^{2}, \quad \operatorname{diam}(M) \leq 2 .
$$

Now consider the product $\left(M \times S^{2}\right)$ so that it satisfies the condition (2.7). It is easy to see that $M \times S^{2}$ is a fibration over $T^{2}$ with fiber $S^{1} \times S^{2}$.

3. Ricci curvature pinching. If one replaces the lower bound on Ricci curvature by pinching and adds the lower volume bound, then one can prove that the second case in Theorem 2.4 does not happen. In [3], Cheeger and Colding extended the splitting theorem of sectional curvature version to that of Ricci curvature version. Namely, the splitting theorem does hold for the limit space of Gromov-Hausdorff convergent sequence each term of which satisfies a diameter upper bound and Ricci condition that $\operatorname{Ric}\left(M_{i}, g_{i}\right) \geq-\epsilon_{i} \rightarrow 0$. Thus, using the abelian covering manifold which gives an extended version of splitting theorem and modifying the argument in [13] a little bit, one can easily prove the following lemma.

LEMMA 3.1. Let $M_{i}$ be a sequence of compact Riemannian $n$-manifolds with $\operatorname{Ric}\left(M_{i}\right) \geq$ $-\epsilon_{i} \rightarrow 0, \operatorname{diam}\left(M_{i}\right)=1, b_{1}\left(M_{i}\right)=b_{1}$, and $\widetilde{M}_{i}$ the universal cover of $M_{i}$. Then, for any $\widetilde{p_{i}} \in \widetilde{M_{i}},\left(\widetilde{M_{i}}, \widetilde{g_{i}}, \widetilde{p_{i}}\right)$ subconverges to $\left(\mathbb{R}^{k} \times X_{0}, x_{0}, d\right)$ in the pointed Gromov-Hausdorff distance, where $k \geq b_{1}$, and $X_{0}$ is a compact length space. 
We would like to remark that the dimension of the Euclidean factor is greater than or equal to the first Betti number.

THEOREM 3.2. Given $\Lambda>0, v>0, D>0$, and a natural number $n$, there exists $\epsilon=\epsilon(\Lambda, v, D, n)>0$ such that if $\left(M^{n}, g\right)$ is a Riemannian $n$-manifold satisfying

$$
\begin{gathered}
\left|K_{g}\right| \leq \Lambda, \quad \operatorname{diam}(M) \leq D, \quad \operatorname{vol}(M, g) \geq v, \\
|\operatorname{Ric}(M)| \leq \epsilon, \quad b_{1}(M)=n-2,
\end{gathered}
$$

then $M$ is an infranilmanifold.

Proof. Suppose the theorem does not hold. Then there are a sequence of positive real numbers $\epsilon_{i} \rightarrow 0$ and a sequence of Riemannian $n$-manifolds $\left(M_{i}, g_{i}\right)$ satisfying (3.1), but $M_{i}$ is not an infranilmanifold for all $i$.

With the volume condition, the standard Cheeger-Gromov compactness theorem [7, $10]$ tells that there exists a subsequence of $\left(M_{i}, g_{i}\right)$ converging to a smooth $n$-manifold with a $C^{1, \alpha}$ Riemannian metric $(M, g)$ in the $C^{1, \alpha^{\prime}}$ topology with $0<\alpha^{\prime}<\alpha$. In particular, $M_{i}$ is diffeomorphic to $M$ for all $i$ sufficiently large. Furthermore, since $\left|\operatorname{Ric}\left(M_{i}, g_{i}\right)\right| \leq$ $\epsilon_{i} \rightarrow 0$, the Ricci equation argument in harmonic coordinates [1] shows that the metric $g$ is, in fact, $C^{\infty}$. Consequently, $\left(M_{i}, g_{i}\right)$ subconverges to a smooth Ricci flat Riemannian manifold $(M, g)$ in the $C^{\infty}$ topology. This, together with the curvature condition, implies that the universal cover $\widetilde{M}_{i}$ converges to the universal cover $\widetilde{M}$ (cf. [5, Theorem 2.7]). Now, applying Lemma 3.1, $\widetilde{M}$ is isometric to $\mathbb{R}^{k} \times X_{0}^{n-k}$ with $k \geq b_{1}(M)=n-2$, where $X_{0}$ is a compact Riemannian manifold. Since $g$ is Ricci-flat, $X_{0}^{n-k}$ is also a Ricci flat manifold. Since $n-k \leq 2, X_{0}^{n-k}$ is a flat manifold, and so $g$ is a flat metric on $M$. Therefore, $M_{i}$ admits a flat metric for $i$ sufficiently large and so does $\widetilde{M}_{i}$.

On the other hand, since $M_{i}$ is not an infranilmanifold, Theorem 2.4 shows that $\widetilde{M}_{i}$ is diffeomorphic to $S^{2} \times \mathbb{R}^{n-2}$. So, $S^{2} \times \mathbb{R}^{n-2}$ admits a flat metric, but this is impossible because of the Cartan-Hadamard theorem. The proof is complete.

REMARK 3.3. In the collapsing case, the same result as Theorem $3.2 \mathrm{might}$ also hold.

ACKNOWLEDGMENT. This work was supported by Grant no. R05-2000-000-00013-0 from the Basic Research Program of the Korea Science and Engineering Foundation.

\section{REFERENCES}

[1] M. Anderson, Convergence and rigidity of manifolds under Ricci curvature bounds, Invent. Math. 102 (1990), no. 2, 429-445.

[2] W. Ballmann, M. Gromov, and V. Schroeder, Manifolds of Nonpositive Curvature, Progress in Mathematics, vol. 61, Birkhäuser Boston, Massachusetts, 1985.

[3] J. Cheeger and T. H. Colding, Lower bounds on Ricci curvature and the almost rigidity of warped products, Ann. of Math. (2) 144 (1996), no. 1, 189-237.

[4] M. Fernández, M. J. Gotay, and A. Gray, Compact parallelizable four-dimensional symplectic and complex manifolds, Proc. Amer. Math. Soc. 103 (1988), no. 4, 1209-1212.

[5] K. Fukaya, Theory of convergence for Riemannian orbifolds, Japan. J. Math. (N.S.) 12 (1986), no. 1, 121-160.

[6] S. Gallot, A Sobolev inequality and some geometric applications, Spectra of Riemannian Manifolds, Kaigai, Tokyo, 1983, pp. 45-55. 
[7] R. E. Greene and H. Wu, Lipschitz convergence of Riemannian manifolds, Pacific J. Math. 131 (1988), no. 1, 119-141.

[8] M. Gromov, Curvature, diameter and Betti numbers, Comment. Math. Helv. 56 (1981), no. 2, 179-195.

[9] _ Groups of polynomial growth and expanding maps, Inst. Hautes Études Sci. Publ. Math. 53 (1981), 53-73.

[10] _ Structures Métriques pour les Variétés Riemanniennes [Metric Structures for Riemann Manifolds], edited by J. Lafontaine and P. Pansu, Textes Mathématiques, vol. 1, CEDIC, Paris, 1981.

[11] J. Kim and G. Yun, Symplecticity of 4-dimensional nil-manifolds and scalar curvature, Bull. Korean Math. Soc. 35 (1998), no. 3, 563-570.

[12] T. Yamaguchi, Manifolds of almost nonnegative Ricci curvature, J. Differential Geom. 28 (1988), no. 1, 157-167.

[13] _ Manifolds of almost nonnegative curvature, J. Math. Sci. (New York) 94 (1999), no. 2, 1270-1310.

[14] G. Yun, A note on the fundamental groups of manifolds with almost nonnegative curvature, Proc. Amer. Math. Soc. 125 (1997), no. 5, 1517-1522.

Gabjin Yun: Department of Mathematics, Myong Ji University, San 38-2, Namdong, Yongin, Kyunggi 449-728, Korea

E-mail address: gabjin@mju.ac.kr 


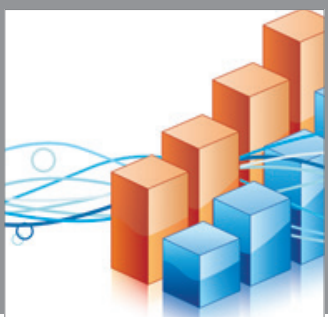

Advances in

Operations Research

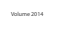

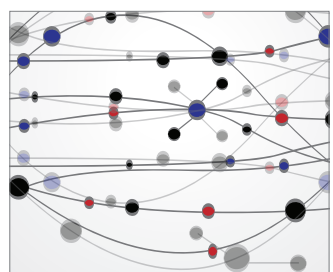

\section{The Scientific} World Journal
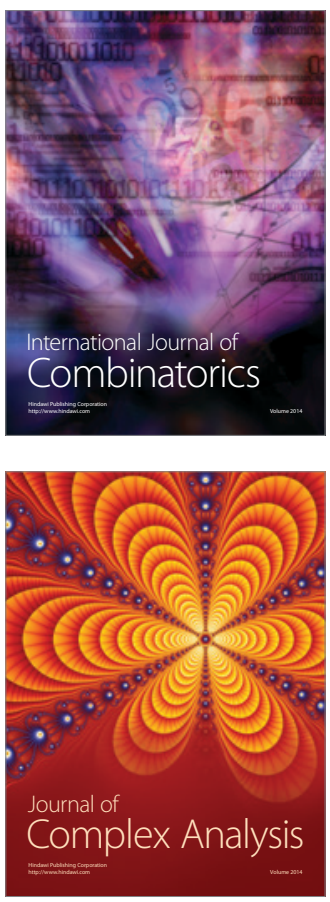

International Journal of

Mathematics and

Mathematical

Sciences
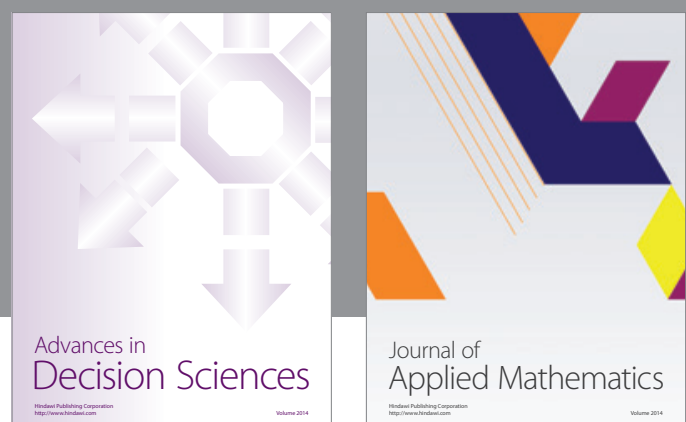

Journal of

Applied Mathematics
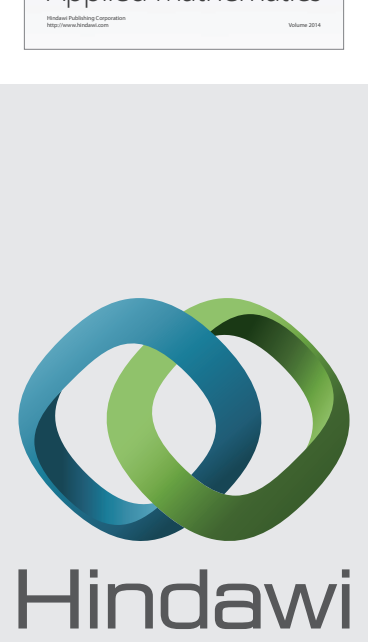

Submit your manuscripts at http://www.hindawi.com
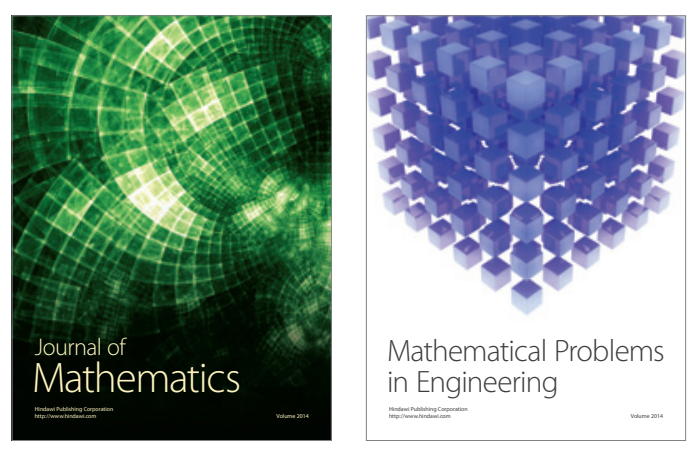

Mathematical Problems in Engineering
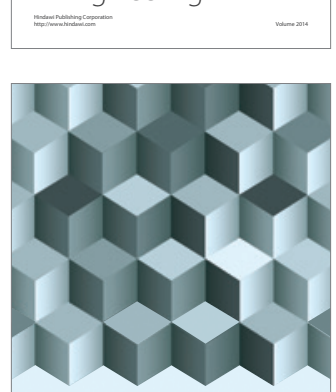

Journal of

Function Spaces
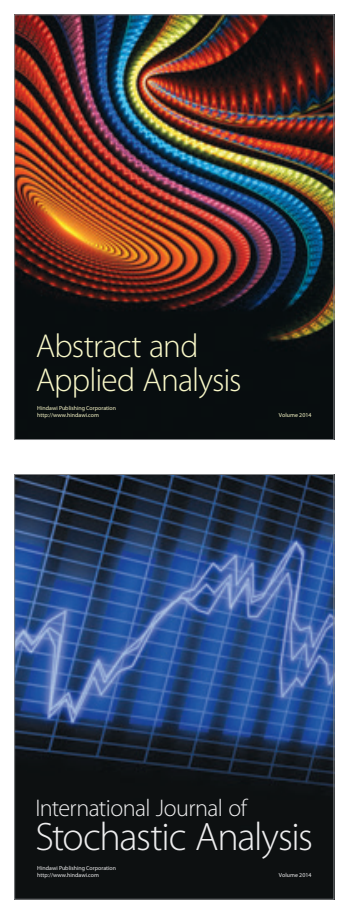

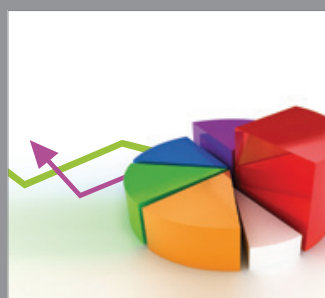

ournal of

Probability and Statistics

Promensencen
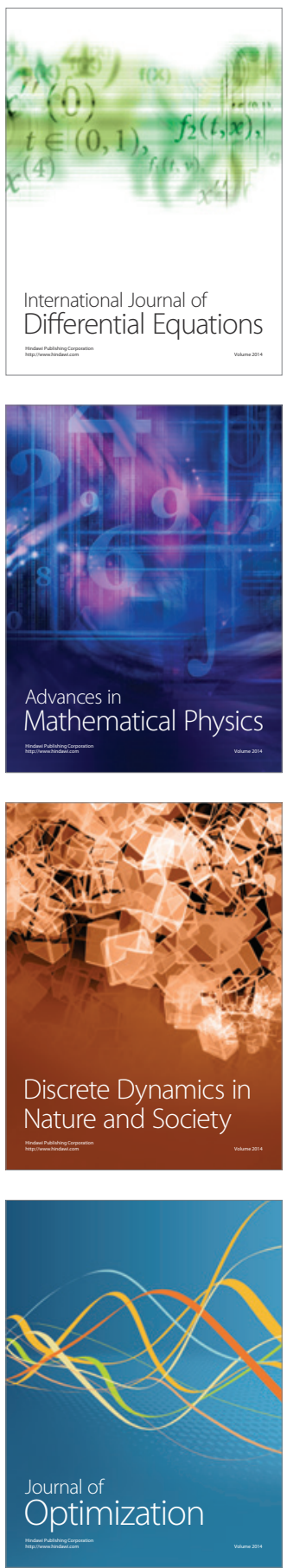